\title{
Inter-Particle Interactions: Energy Potentials, Energy Transfer, and Nanoscale Mechanical Motion in Response to Optical Radiation
}

\author{
David S. Bradshaw and David L. Andrews \\ School of Chemistry, University of East Anglia, Norwich Research Park, \\ Norwich NR4 7TJ, U. K.
}

\begin{abstract}
In the interactions between particles of material with slightly different electronic levels, unusually large shifts in the pair potential can result from photo-excitation, and on subsequent electronic excitation transfer. To elicit these phenomena it is necessary to understand the fundamental differences between a variety of optical properties deriving from dispersion interactions, and processes such as resonance energy transfer that occur under laser irradiance. This helps dispel some confusion in the recent literature. By developing and interpreting the theory at a deeper level, it can be anticipated that in suitable systems, light absorption and energy transfer will be accompanied by significant displacements in inter-particle separation, leading to nanoscale mechanical motion.
\end{abstract}

Keywords: Dispersion interactions, optical binding, optical forces, optomechanics, light absorption, quantum electrodynamics 


\section{Introduction}

The presence of electromagnetic interactions between molecules, or other particles, can elicit two clearly different types of observable, distinguished by whether or not there is an overall movement of energy from one site to the other. A raft of energy transfer interactions is associated with cases where there is such a relocation of energy: conversely, electromagnetic interactions without energy displacement are responsible for a pair potential energy, and lead to an inter-particle force. Such forces are nonetheless generally influenced by the electronic state of the materials of which they are composed; although ground-state interactions are most common it is possible, through optical excitation, to engineer changes in any interaction potential. With suitably designed systems, throughput laser radiation can thus produce nanoscale mechanical motion. To elicit the detailed mechanical behavior associated with such effects, and to exploit the interplay between optical properties deriving from dispersion interactions and processes such as resonance energy transfer (RET) occurring under laser irradiance, it is first necessary to understand key differences in their physical origin. Putting the theory on a sound basis, and helping to dispel some confusion in the recent literature, a specific scheme that deploys a combination of processes and interactions may then be introduced.

Starting from an initial focus on the simple mechanisms of interacting permanent dipoles and RET, we present a quantum formulation of the dispersion interaction, ${ }^{1-6}$ the intrinsic coupling between particles which is always attractive when both particles are in their ground state. Nonetheless, as will be shown, the associated force may become repulsive if one interacting particle is electronically excited. We then address laser-induced optical binding, ${ }^{7-12}$ where the coupling potential between the particles is modified due to an off-resonant throughput beam, 
and laser-assisted resonance energy transfer, ${ }^{13-15}$ in which RET is itself influenced by the input laser. Although similar in nature from a theoretical perspective, each of these phenomena can be divided into distinct categories that, in practice, correspond to different physical observables - relating either to a potential energy (for a system with identical initial and final states) or a transfer rate (where initial and final states differ). The following Sections $2-4$ first lay down the general principles and then the applications to the cases detailed above. In Section 5, the possibility of developing these optical interactions in tandem is examined with an illustrative example, and a final discussion concerning technical details of measurement and possible applications concludes the paper.

\section{Quantum framework}

To most accurately describe the particle-light interactions responsible for the observables introduced in the previous Section demands a quantum theoretical outlook. ${ }^{16-18}$ When employing a quantum formalism, such interactions are most conveniently visualized using the graphical aids known as Feynman diagrams. These pictorial representations depict and assist the mathematical formulation, facilitating the visualization and comprehension of each phenomenon. Such diagrams, whose origin is in the realm of elementary particle physics, are now widely used for the representing the interactions of larger particles - molecules, quantum dots or other sub-micron Rayleigh particles: see for example ref. [18]. In each such application, since the electrons are bound to the nuclei, the particles assume a stationary center-of-mass position (depicted by vertical lines in the diagrams) and electromagnetic radiation is viewed as propagating about them (signified by wavy lines); the intersection of the lines denotes particle- 
light interactions. These representations will play an important role in not only the visualization of particle-light interactions, but enable an understanding of the fundamental differences between a variety of optical properties and determine their key physical origins.

The general principles of the quantum framework now follow, utilization of which will result in a mathematical description of each observable in Sections 3 and 4, and their corresponding Feynman diagrams will also be presented. A complete quantum study is given, although intricate details of the derivations are kept to a minimum. As a starting point for the analysis, using the Power-Zienau-Woolley approach, ${ }^{19}$ the Hamiltonian energy for a system comprising particles labeled $\xi$ is promoted to operator status producing an operator, $H$, which in multipolar form is exactly expressible as;

$H=\sum_{\xi} H_{\mathrm{part}}(\xi)+\sum_{\xi} H_{\mathrm{int}}(\xi)+H_{\mathrm{rad}}=H_{0}+\sum_{\xi} H_{\mathrm{int}}(\xi)$

where $H_{\text {part }}(\xi)$ is the Hamiltonian for particle $\xi, H_{\text {rad }}$ is the radiation Hamiltonian and $H_{\text {int }}(\xi)$ is the Hamiltonian representing the interaction of the radiation field with $\xi$. For reasons that will emerge, it is important that there is no term directly coupling different particles; this signifies that any interaction between the latter has to engage the coupling each has with the radiation field. In detail, $H_{\text {int }}(\xi)$ acts as a perturbation to the system that is initially in the stationary eigenstate of $H_{0}$, given by Eq. (1) excluding $H_{\text {int }}(\xi)$. This eigenstate basis, $|\psi\rangle$, forms a composite set expressible in the following form; 
$|\psi\rangle=\left|\psi_{\text {part }}\right\rangle\left|\psi_{\text {rad }}\right\rangle \equiv\left|\psi_{\text {part }} ; \psi_{\text {rad }}\right\rangle$.

Here, $\left|\psi_{\text {part }}\right\rangle$ defines the status of all the particles comprising a product of state vectors for each particle $\xi$, each with associated energy $E_{\psi}(\xi) ;\left|\psi_{\text {rad }}\right\rangle$ represents radiation (number) states. One of the first notable differences from a semiclassical formulation is that there is no circumstance where the basis states (2) are eigenstates of the full Hamiltonian (1), due to the presence of the interaction operator term. (In a semiclassical formulation, that operator is zero if there is no light present). Consequently, the system energy is not identifiable with the sum of the energies $E_{\psi}(\xi)$ - in particular, pair interactions have to engage the quantum radiation field.

In the electric-dipole approximation, the $H_{\text {int }}(\xi)$ operator may be decomposed into the scalar product of the transverse electric displacement field, $\mathbf{d}^{\perp}(\mathbf{R})$, (the radiation operator) and the electric dipole moment operator, $\boldsymbol{\mu}$, (the particle operator) - the full expression is written;

$$
H_{\mathrm{int}}(\xi)=-\varepsilon_{0}^{-1} \sum_{\xi} \boldsymbol{\mu}_{\xi} \cdot \mathbf{d}^{\perp}\left(\mathbf{R}_{\xi}\right)
$$

Here, the dipole moment operator, $\boldsymbol{\mu}_{\xi}$, operates on particle states, $\left|\psi_{\text {part }}\right\rangle$, and the transverse electric field operator, $\mathbf{d}^{\perp}\left(\mathbf{R}_{\xi}\right)$, operates on $\left|\psi_{\text {rad }}\right\rangle$. The latter involves a summation over all wave-vectors, $\mathbf{k}$, and polarizations, $\eta$, and is usually written as the following mode expansion; 
$\mathbf{d}^{\perp}\left(\mathbf{R}_{\xi}\right)=\mathrm{i} \sum_{\mathbf{k}, \eta}\left(\frac{\hbar c k \varepsilon_{0}}{2 V}\right)^{1 / 2}\left\{\mathbf{e}_{\mathbf{k}}^{(\eta)} a_{\mathbf{k}}^{(\eta)} e^{\mathrm{i}\left(\mathbf{k} \cdot \mathbf{R}_{\xi}\right)}-\overline{\mathbf{e}}_{\mathbf{k}}^{(\eta)} a_{\mathbf{k}}^{\dagger(\eta)} e^{-\mathrm{i}\left(\mathbf{k} \cdot \mathbf{R}_{\xi}\right)}\right\}$

where $\mathbf{e}_{\mathbf{k}}^{(\eta)}$ is the polarization unit vector $\left(\overline{\mathbf{e}}_{\mathbf{k}}^{(\eta)}\right.$ being its complex conjugate), $V$ is an arbitrary quantization volume and $a_{\mathbf{k}}^{(\eta)}, a_{\mathbf{k}}^{\dagger(\eta)}$ are respectively the photon annihilation and creation operators for a mode $(\mathbf{k}, \eta)$. The latter operators act on the radiation states through $a_{\mathbf{k}}^{(\eta)}\left|n_{\mathbf{k}, \eta}\right\rangle=n^{\frac{1}{2}}\left|(n-1)_{\mathbf{k}, \eta}\right\rangle$ and $a_{\mathbf{k}}^{\dagger(\eta)}\left|n_{\mathbf{k}, \eta}\right\rangle=(n+1)^{\frac{1}{2}}\left|(n+1)_{\mathbf{k}, \eta}\right\rangle ;$ the appearance of these operators in $H_{\text {int }}$ represent photon creation and annihilation.

The matrix element $M_{F I}$ which signifies the coupling between initial and final states containing information on the particle and radiation - is determined by time-dependent perturbation theory. ${ }^{20}$ The result is cast, using the resolvent operator formalism, as the following infinite series for elements of a matrix operator $M$;

$\left(M_{F I}\right)_{\mathrm{sys}} \equiv\langle F|M| I\rangle=\left\langle F\left|\sum_{p=0}^{\infty} H_{\mathrm{int}}\left(T_{0} H_{\mathrm{int}}\right)^{p}\right| I\right\rangle \quad$,

where the subscript 'sys' clearly defines the use of full system rather than particle states, $|I\rangle$ and $|F\rangle$ represent the initial and final states, respectively, and generally the interaction Hamiltonian, $H_{\text {int }}$, acts upon both particle and radiation components of the system 
state. Moreover $T_{0} \approx\left(E_{I}-H_{0}\right)^{-1}$, where $E_{I}$ is the energy of the initial state. Implementing the completeness relation $\sum_{J}|J\rangle\langle J|=1$ gives:

$$
\begin{aligned}
\left(M_{F I}\right)_{\mathrm{sys}}= & \left\langle F\left|H_{\mathrm{int}}\right| I\right\rangle+\sum_{R} \frac{\left\langle F\left|H_{\mathrm{int}}\right| R\right\rangle\left\langle R\left|H_{\mathrm{int}}\right| I\right\rangle}{\left(E_{I}-E_{R}\right)}+\sum_{R, S} \frac{\left\langle F\left|H_{\mathrm{int}}\right| S\right\rangle\left\langle S\left|H_{\mathrm{int}}\right| R\right\rangle\left\langle R\left|H_{\mathrm{int}}\right| I\right\rangle}{\left(E_{I}-E_{R}\right)\left(E_{I}-E_{S}\right)} \\
& +\sum_{R, S, T} \frac{\left\langle F\left|H_{\mathrm{int}}\right| T\right\rangle\left\langle T\left|H_{\mathrm{int}}\right| S\right\rangle\left\langle S\left|H_{\mathrm{int}}\right| R\right\rangle\left\langle R\left|H_{\mathrm{int}}\right| I\right\rangle}{\left(E_{I}-E_{R}\right)\left(E_{I}-E_{S}\right)\left(E_{I}-E_{T}\right)}+\ldots
\end{aligned}
$$

Here, the intermediate virtual states of the particle are denoted by $|R\rangle,|S\rangle,|T\rangle \ldots$ upon which operates $H_{0}$ to deliver energy eigenvalues $E_{R}, E_{S}, E_{T}$, etc. The interaction Hamiltonian is linear in the electromagnetic fields, which have corresponding mode expansions that are linear in the photon annihilation and creation operators. Therefore, the $j^{\text {th }}$ term in Eq. (6) delivers the leading contribution to the matrix element for any process involving $j$ particle-photon events.

Returning to Eq. (5), it is clear that when the initial and final system states are the same (such as for optical binding and dispersion interactions: details are given later), then only diagonal elements of the matrix element will arise. Explicitly, for a system state $|I\rangle$, and for conciseness writing $\left\langle\left(M_{I I}\right)_{\text {sys }}\right\rangle\left(\mathbf{R}_{1}, \mathbf{R}_{2}, \ldots\right) \equiv\langle M\rangle_{\text {sys }}\left(\left\{\mathbf{R}_{\xi}\right\}\right)$, the result is expressible as;

$$
\langle M\rangle_{\text {sys }}\left(\left\{\mathbf{R}_{\xi}\right\}\right) \equiv\langle I|M| I\rangle\left(\left\{\mathbf{R}_{\xi}\right\}\right)=\prod_{m, \mathbf{k}, \eta}\left\langle\varphi_{\xi_{m}}\left|\left\langle n_{\mathbf{k}, \eta}|M| n_{\mathbf{k}, \eta}\right\rangle\right| \varphi_{\xi_{m}}\right\rangle\left(\left\{\mathbf{R}_{\xi}\right\}\right)
$$


where $\varphi_{\xi_{m}}$ is the wavefunction of each specific particle $\xi_{m}$ and the dependence on the set $\left\{\mathbf{R}_{\xi}\right\}$ , i.e. the position of all the particles, is now included. For this case, there is no transfer of energy from the particles to or from the radiation field, nor is any directly transferred between the particles. The diagonal elements of (5) thus represent expectation values with respect to the operator $M$, arising due to the interaction Hamiltonian acting as a perturbation on members of the basis state set in which the particles are uncoupled. Under these conditions, and because of the nonlinear dependence on $H_{\text {int }}$ that is entailed, the expectation value $\langle M\rangle_{\text {sys }}\left(\left\{\mathbf{R}_{\xi}\right\}\right)$ represents an energy contribution that depends on the relative spatial displacements of the component particles, physically representing their electrodynamic coupling. Accordingly it is expedient to regard the energy terms $E_{\psi}(\xi)$ as individual electronic energies, and $\langle M\rangle_{\text {sys }}\left(\left\{\mathbf{R}_{\xi}\right\}\right)$ as a potential energy. Since each pair energy shift is registered with respect to the sum energies of two isolated components, i.e. the system energy at infinite separation, it can be interpreted as the potential energy of the pair. This type of calculation is timeindependent, since not only the state vectors $\left|\psi_{\text {part }}\right\rangle$ but also the radiation state vectors $\left|\psi_{\text {rad }}\right\rangle$ are identical in the initial and final states, and therefore of necessity an identical number of photon creation and annihilation operators - as appear in Eq. (4) - are always employed. Resulting from this, it is apparent that only even-order terms arise in Eq. (6), since matching a creation operator with an annihilation operator necessarily involves even number of photons. This tallies with the fact that in the interaction representation the photon creation operator carries a temporal phase factor $e^{\mathrm{i} \omega t}$ and the annihilation operator, $e^{-\mathrm{i} \omega t}(t$ denotes time and $\omega$ is the angular frequency $)^{21}$ - so that unless both have the same number of appearances, the associated matrix element contribution will have an unphysically rapid oscillation about zero. 
Analogously, in the semi-classical approach there would have to be a matching number of terms involving the positive frequency and negative frequency components of the electric field. The mechanical action of such interacting systems is now apparent following the calculation for a force $^{22}$ arising between particles $\xi_{q}$ and $\xi_{r}$ :

$\mathbf{F}\left(\xi_{q}, \xi_{r}\right)=-\frac{\partial\langle M\rangle_{\mathrm{sys}}\left(\left\{\mathbf{R}_{\xi}\right\}\right)}{\partial\left(\mathbf{R}_{\xi_{q}}-\mathbf{R}_{\xi_{r}}\right)}$.

Alternatively, when $|I\rangle$ and $|F\rangle$ differ, then off-resonant elements are observed, and the matrix elements $M_{F I}$ represent perturbations due to the interaction Hamiltonian acting on unperturbed eigenstates of $H_{0}$. This is the case for coupling interactions such as resonance energy transfer and laser-assisted resonance energy transfer (detailed later). From the experimental point of view, the consequence of such energy relocation processes, which involve a clearly time-dependent evolution of the final state from the initial, is that there is an identifiable and experimentally determinable rate at which the energy transfers. Hence, the observable transfer rate is the physical quantity to be established from the calculations, normally derived from $M_{F I}$ via the Fermi Rule. ${ }^{23}$

To complete this overview of founding principles, it is worth clarifying the status of the separation between electronic and internal nuclear degrees of freedom. The $\mathbf{R}_{\xi}$ vectors featured in the above analysis denote center-of-mass positions, following the conventional separation of internal vibrational and orientational degrees of freedom. Moreover in the systems of interest here, the latter motions are treated classically, with conventional isotropic 
averages utilized where necessary to account for free rotation. It remains to effect the usual Born-Oppenheimer partition of the particle states $\left|\psi_{\text {part }}\right\rangle$ into electronic and vibrational parts, $\left|\varphi_{\xi_{m}}\right\rangle=\left|\psi_{\xi_{m}}^{\text {elec }}\right\rangle\left|\Psi_{\xi_{m}}^{\text {nuc }}\right\rangle$, with a corresponding additivity of energies, $E_{\xi_{m}}=E_{\xi_{m}}^{\text {elec }}+E_{\xi_{m}}^{\text {nuc }}$. The nuclear wavefunction will generally factor into components for each vibrational mode, the corresponding energies again being additive. Importantly, according to the Franck-Condon Principle, static and transition dipole moments factorize in the following sense:

$$
\boldsymbol{\mu}^{p q\left(\xi_{m}\right)} \equiv\left\langle\Psi_{\xi_{m: p}}^{P(\text { nuc })}\left|\left\langle\psi_{\xi_{m}}^{p(\text { elec })}|\hat{\boldsymbol{\mu}}| \psi_{\xi_{m}}^{q(\mathrm{elec})}\right\rangle\right| \Psi_{\xi_{m \cdot q}}^{Q(\mathrm{nuc})}\right\rangle \cong\left\langle\Psi_{p}^{P(\text { nuc })} \mid \Psi_{q}^{Q(\mathrm{nuc})}\right\rangle_{\xi_{m}}\left\langle\psi^{p}|\hat{\boldsymbol{\mu}}| \psi^{q}\right\rangle_{\xi_{m}}
$$

The first term on the right-hand side of Eq. (9) is in general a product of Franck-Condon factors for each vibrational mode. Thus, each dipole moment connecting states $p$ and $q$ has an implicit dependence on the associated vibrational states, indicated by $P, Q$ above. Where appropriate, we shall draw on the implications of Born-Oppenheimer separability in the following.

\section{Lowest-order interactions}

\subsection{Permanent dipole coupling}

The simplest interactions involving two particles are those deriving from permanent dipole couplings, namely through electrostatic interactions. In terms of quantum electrodynamics, such couplings are mediated by virtual photons created at particle $\xi_{1}$ and subsequently 
annihilated at particle $\xi_{2}$, and vice-versa - see the Feynman diagram of Fig. 1(a). This phenomenon entails two particle-photon interactions and, as a result, corresponds to the second term of Eq. (6) in a second-order perturbation. These inter-particle interactions produce a potential energy across the pair (an energy shift $\Delta E$ ), the calculation leading to the familiar expression from Coulomb's Law, i.e.;

$\Delta E=\mu_{i}^{00\left(\xi_{1}\right)} V_{i j}(0, \mathbf{R}) \mu_{j}^{00\left(\xi_{2}\right)}=\frac{\mu_{i}^{00\left(\xi_{1}\right)} \mu_{j}^{00\left(\xi_{2}\right)}}{4 \pi \varepsilon_{0} R^{3}}\left(\delta_{i j}-3 \hat{R}_{i} \hat{R}_{j}\right)$,

where Einstein summation is employed, $\boldsymbol{\mu}^{00\left(\xi_{m}\right)}$ is the permanent (static) dipole moment of particle $m$, and the generalized coupling tensor, $V_{i j}(k, \mathbf{R})$, is expressed as, ${ }^{24,25}$

$V_{i j}(k, \mathbf{R})=\frac{e^{\mathrm{i} k R}}{4 \pi \varepsilon_{0} R^{3}}\left((1-\mathrm{i} k R)\left(\delta_{i j}-3 \hat{R}_{i} \hat{R}_{j}\right)-(k R)^{2}\left(\delta_{i j}-\hat{R}_{i} \hat{R}_{j}\right)\right)$

Here, $\mathbf{R} \equiv \mathbf{R}_{\xi_{2}}-\mathbf{R}_{\xi_{1}}$, also expressible as $\mathbf{R}=\hat{\mathbf{R}} R$, is the displacement vector between $\xi_{1}$ and $\xi_{2}, \hbar c k$ is the energy transferred and $e^{\mathrm{i} k R}$ is a phase factor. The lack of energy transferral between the particles explains the calculation of an energy shift (potential energy), rather than the determination of a transfer rate; the latter will be seen in the following sub-section.

\subsection{Resonance energy transfer}


The simplest transfer mechanism between two neutral particles, i.e. without the presence of permanent dipoles, is termed resonance energy transfer (RET) ${ }^{26-31}$ This process represents a transport of electronic excitation based on transition dipole-dipole coupling. In detail, if any neighboring particle in the vicinity of a directly photo-excited, electronically activated center has a suitably disposed electronic state, of a similar or slightly lower energy, that neighbor may acquire the major part of the initial electronic excitation through RET. ${ }^{32,33}$

Consider the pairwise transfer of excitation between two particles $\xi_{1}$ and $\xi_{2}$, which denote donor and acceptor, respectively, in these transfer systems. Prior excitation of the donor generates an electronically excited particle $\xi_{1}^{*}$, and release of the energy is accompanied by donor decay to the ground electronic state. Acquiring the energy, $\xi_{2}$ undergoes a transition from its ground to its excited state, $\xi_{2}^{*}$. In terms of quantum electrodynamics, RET is again interpreted as the creation of a virtual photon at $\xi_{1}$ and its annihilation at $\xi_{2}$ and vice-versa; the inclusion of both is required to allow for energy uncertainty considerations. This mechanism again corresponds to the second term of Eq. (6) through its two particle-photon interactions - a key difference to the previous interaction is that the initial and final states of the two particles change, Fig. 1(b).

The intricate derivation of $M_{F I}$ for RET is given elsewhere, ${ }^{24}$ the result of the calculations are as follows;

$M_{F I}=\mu_{i}^{0 \alpha\left(\xi_{1}\right)} V_{i j}(k, \mathbf{R}) \mu_{j}^{\beta 0\left(\xi_{2}\right)}$, 
where $V_{i j}(k, \mathbf{R})$ is given by Eq. (11), $\boldsymbol{\mu}^{0 \alpha\left(\xi_{1}\right)}$ corresponds to a transition from an excited state $\alpha$ to ground state 0 within the donor, and $\mu^{\beta 0\left(\xi_{2}\right)}$ a transition from the ground to an excited state $\beta$ in the acceptor - these are transition dipole moments rather than the previous permanent dipoles. On inspection of Eq. (12) via (11), it is evident that the first term is dominant in the short-range or near-zone region ( $k R<<1$ ), and the third term in the long-range or wave-zone ( $k R>>1$ ). From Eq. (12), the transfer rate $\Gamma$ is determined from the Fermi Golden Rule, which is expressible as; ${ }^{23}$

$\Gamma=\frac{2 \pi}{\hbar}\left|M_{F I}\right|^{2} \rho_{F}$

where $\rho_{F}$ is the density of acceptor final states. For a system of freely rotating dipoles, implementation of rotational averaging on Eq. (13) is required - this delivers the result;

$\Gamma \sim \frac{1}{9}\left|\boldsymbol{\mu}^{0 \alpha\left(\xi_{1}\right)}\right|^{2}\left|\boldsymbol{\mu}^{\beta 0\left(\xi_{2}\right)}\right|^{2} A(k, R)$,

where the excitation transfer function, $A(k, R)$, is defined by;

$$
A(k, R)=V_{i j}(k, \mathbf{R}) \bar{V}_{i j}(k, \mathbf{R})=\frac{2}{\left(4 \pi \varepsilon_{o} R^{3}\right)^{2}}\left(3+(k R)^{2}+(k R)^{4}\right)
$$


From this equation, where the initial and final terms dominate in the respective short- and longrange regions as Eq. (12), both the $R^{-6}$ dependence of the Förster result and the $R^{-2}$ dependence of inverse-square laws are evident. The presence of these terms (and the distinctive middle term) in a single expression signifies that the two processes are the short- and long-range asymptotes of a unified theory. ${ }^{25}$ Furthermore, it has been shown elsewhere ${ }^{34}$ that the inclusion of features associated with the vibrational continua of the donor and acceptor states leads to a dependence on spectral overlap, whose detailed form differs from term to term in Eq. (15). Such features are responsible for the spectroscopic gradient that typically ensures directionality of energy flow between particles. ${ }^{35}$

\section{Higher-order interactions}

\subsection{Dispersion interaction}

Replacing the permanent dipoles of Section 2.1 with a pair of neutral, non-polar particles means that electrostatic dipolar interactions are no longer present. These types of particle interact through a weaker, higher-order interaction termed the dispersion interaction. Classically, this interaction engages the induction of a transient dipole within one particle by the fluctuation of electric charge in the other and vice-versa. The quantum theory of dispersion interactions first

derived by London, characterized by an $R^{-6}$ dependence, is valid over short-range distances; an extension by Casimir and Polder gives an $R^{-7}$ dependence over large distances. ${ }^{36}$ With our present focus on particles in close proximity, the following expression is valid for the short-range only. 
In terms of quantum electrodynamics, dispersion interactions entail two virtual photons producing four particle-photon couplings, Fig. 2(a). Hence, a fourth-order perturbation corresponding to the fourth term of Eq. (6). Employing the latter the following general expression is ascertained;

$\Delta E=-\sum_{s, t} \frac{\boldsymbol{\mu}_{i}^{a s\left(\xi_{1}\right)} V_{i j}(0, \mathbf{R}) \boldsymbol{\mu}_{j}^{b t\left(\xi_{2}\right)} \boldsymbol{\mu}_{k}^{s a\left(\xi_{1}\right)} V_{k l}(0, \mathbf{R}) \boldsymbol{\mu}_{l}^{t b\left(\xi_{2}\right)}}{E_{s a}^{\xi_{1}}+E_{t b}^{\xi_{2}}}$,

where $a \in\{0, \alpha\}, b \in\{0, \beta\}$ and $E_{s a}=E_{s}-E_{a}$ etc.; moreover, $s$ and $t$ are intermediate virtual states of particles $\xi_{1}$ and $\xi_{2}$, respectively. On applying an isotropic average;

$\Delta E=-\frac{1}{24 \pi^{2} \varepsilon_{0}{ }^{6} R^{6}} \sum_{s, t} \frac{\left|\boldsymbol{\mu}^{a s\left(\xi_{1}\right)}\right|^{2}\left|\boldsymbol{\mu}^{b t\left(\xi_{2}\right)}\right|^{2}}{E_{s a}^{\xi_{1}}+E_{t b}^{\xi_{2}}}$,

which reduces to the London formula when $a$ and $b$ denote ground levels. A consequence of such constraints is that $E_{s a}^{\xi_{1}}$ and $E_{t b}^{\xi_{2}}$ always produce positive numbers, invariably creating a negative quantity for the result of Eq. (17). Physically, this negative result, with a negative index for the power of $R$, corresponds to an attractive interaction. For particles in their ground state, this is always the case. Alternatively, when either (or both) particles are electronically excited through direct absorption, or via RET, the dispersion interaction may become repulsive; ${ }^{3}$ this is discussed further in Section 5. 


\subsection{Optical binding}

The phenomenon of optical binding occurs between interacting neutral particles under the influence of throughput radiation. In detail, this entails an elastic forward-scattering of a passive (off-resonant laser) beam, without any net absorption or stimulated emission - see Fig. $2(b)$ - which leads to modifications to the dispersion interactions. These optically induced interparticle interactions give rise to forces and torques, usually described as optical binding although the forces are not necessarily attractive in form, which are the subject of particularly interesting recent research. ${ }^{10,37-45}$ The phenomenon has increasingly been advocated as a tool for the optical manipulation and configuration of particles, and many optically induced arrays have been observed experimentally..$^{8,46}$

In optical binding, particles $\xi_{1}$ and $\xi_{2}$ both begin and end in their ground states. Four possible routes between the initial and final states arise: photon absorption at $\xi_{1}$ and photon emission, of the same mode, at $\xi_{2}$; the mirror case where $\xi_{1}$ and $\xi_{2}$ are interchanged, and another two cases which involve the static form of contribution. Since centrosymmetric (and therefore necessarily non-polar) particles are under consideration here, static contributions are not presented. Again based on a fourth-order perturbation, corresponding to the fourth term of Eq. (6), the resulting optically induced energy shift between the interacting particles emerges, over all inter-particle displacements, as; 


$$
\begin{aligned}
\Delta E & =\left(\frac{I}{2 \varepsilon_{0} c}\right) e_{i}^{(\eta)} \bar{e}_{l}^{(\eta)} \operatorname{Re}\left(\alpha_{i j}^{a a\left(\xi_{1}\right)}(\omega) V_{j k}(q, \mathbf{R}) \alpha_{k l}^{b b\left(\xi_{2}\right)}(\omega) e^{-i \mathbf{q} \cdot \mathbf{R}}\right. \\
& \left.+\alpha_{i j}^{b b\left(\xi_{2}\right)}(\omega) V_{j k}(q, \mathbf{R}) \alpha_{k l}^{a a\left(\xi_{1}\right)}(\omega) e^{i \mathbf{q} \cdot \mathbf{R}}\right)
\end{aligned}
$$

where $\hbar c q \equiv \hbar \omega$ is the energy per photon of the input beam, $\alpha_{i j}^{a a\left(\xi_{m}\right)}(\omega)$ and $\alpha_{i j}^{b b\left(\xi_{m}\right)}(\omega)$ represents the polarizability tensor of particle $m$ at circular frequency $\omega$; the cases where $a$ or $b$ denote ground levels produces the tensor $\alpha_{i j}^{00\left(\xi_{m}\right)}(\omega)$, as applies in conventional optical binding. ${ }^{11}$ Moreover, although it appears that energy is transferred between the particles in this case, this is an energy that derives solely from the input beam, being held only during the passage of light through the coupled pair - the initial and final system states are still identical. In contrast to Eqs (16) and (17), the above result for the laser-induced interaction is not a monotonic function of distance; energy minima arise. The optical binding force is determined from Eq. (18) via an expression similar to Eq. (8). The magnitude of each pair optical binding force has an $R^{-4}$ dependence in the short-range region, as determined from such a force equation.

Following the optical binding process, which is described by forward Rayleigh scattering, it seems natural to consider the non-forward case where the energy states of the two particles also remain unchanged. Despite this, in terms of quantum electrodynamics, the initial and final states differ slightly as the emitted photon emerges in a different radiation mode to the absorbed photon. Therefore these results, leading to a physically determinable rate, are unexpectedly found in the form of $M_{F I}$. This may be thought of as an anomaly, but is explained by integrating over the direction for the output radiation mode $\left(\mathbf{k}^{\prime}, \eta^{\prime}\right)$ giving a null result for 
$M_{F I}$. Hence, non-forward Rayleigh scattering yields a vanishing optically induced energy shift and thus no associated pairwise optical forces. ${ }^{47}$ The calculation serves to demonstrate that these energies are produced by the evaluation of expectation values between radiation states that are identical not only in energy, but also in linear momentum.

\subsection{Laser-assisted resonance energy transfer}

In addition to optical binding, passive laser control may also enable higher-order effects based on fundamental processes. This can be achieved by engagement of the off-resonant beam, resulting in nonlinear interactions. In the case of RET, a passive laser beam may enhance (or diminish) the process through a conferred optical response known as laser-assisted resonance energy transfer. This again involves elastic forward-scattering of the passive beam, without any net absorption or stimulated emission - as given by Fig. 2(c) - although this is subtly dissimilar to optical binding since initial and final system states now differ. The matrix element again arises from the fourth term of Eq. (6), since the process involves four photon-particle events, and then the transfer rate is found via Eq. (13), giving;

$$
\begin{aligned}
\Gamma & =\left(\frac{\pi I^{2}}{2 \varepsilon_{0}^{2} c^{2} \hbar}\right) \mid e_{i}^{(\eta)} \bar{e}_{l}^{(\eta)}\left(\alpha_{i j}^{0 \alpha\left(\xi_{1}+\right)}(\omega) V_{j k}(k+q, \mathbf{R}) \alpha_{l k}^{\beta 0\left(\xi_{2}-\right)}(-\omega) e^{-i \mathbf{q} \cdot \mathbf{R}}\right. \\
& \left.+\alpha_{i j}^{\beta 0\left(\xi_{2}+\right)}(\omega) V_{j k}(k-q, \mathbf{R}) \alpha_{l k}^{0 \alpha\left(\xi_{1}-\right)}(-\omega) e^{i \mathbf{q} \cdot \mathbf{R}}\right)\left.\right|^{2}
\end{aligned}
$$


where $\alpha_{i j}^{a b\left(\xi_{m} \pm\right)}(\omega)$ represents the transition polarizability tensor of particle $m$, explicitly given in ref. [13], where the frequency argument refers to the local scattering input or output according to the sign in the preceding superscript being positive or negative, respectively. The leading term in the short-range region has an $R^{-6}$ dependence. It is clear, on comparison of Eqs (18) and (19), that both expressions have the same tensor form and phase factors, but the state indices on the final polarizability tensor (in both terms) are interchanged, reflecting the fact that the conventional polarizability is index-symmetric while the transition polarizability is not. The two equations also differ in that only $\hbar c q$, i.e. the energy per photon of the input beam, is involved for optical binding but, additionally, the energy of an initial excitation $\hbar c k$ is a key factor in laser-assisted resonance energy transfer mechanisms. Furthermore, as a result of one expression denoting an energy shift and the other a transfer rate, Eq. (18) is linear in I whereas Eq. (19) is quadratic.

Having the ability to exert optical control over the migration of energy provides a basis for all-optical switching in an adapted form of laser-assisted energy transfer, where the spontaneous process is completely disabled. ${ }^{14,15,48-50}$ This can be achieved in an arrangement where $\mu_{i}^{0 \alpha\left(\xi_{1}\right)} \perp \mathbf{R} \perp \mu_{j}^{\beta 0\left(\xi_{2}\right)}$, which results in spontaneous energy transfer being excluded by geometry. Alternatively, spontaneous transfer is inhibited when the electronic transition in either the donor or acceptor is one-photon forbidden, but two-photon allowed. In either case, switching action induced by the transport of energy is activated only when the passive beam is 'on'. The parallel-processing capability of such a device introduces a variety of applications. For example, it indicates that pixel-based images, written by donor excitation, might be controllably transferred with high fidelity to an acceptor film. 


\section{Observables in tandem}

In the previous analysis, the outline theory of each phenomenon operating in isolation has been given; we now consider two of these interactions in tandem to produce nanoscale mechanical motion via photo-excitation and electronic excitation transfer. Such a combination involves an amalgamation of dispersion interactions and RET. Although operating independently, the presence of energy transfer enables electronic excitation of an interacting particle, resulting in a modification of the potential energy for the dispersion interactions - thus facilitating mechanical motion. As noted earlier, the observables (and the others that follow) for these phenomena remain independent and do not - indeed cannot - together forge a new mechanism, as has occasionally been suggested: we return to this aspect in the Discussion. Other factors must be considered on application of an off-resonant laser of sufficient intensity. Namely, through the laser-assisted resonance energy transfer mechanism of Section 4.3, the transfer rate of RET may be modified, and the likely presence of optical binding (Section 4.2) will itself tend to further modify the extent of nanoscale motion.

Figure 3 visually illustrates, and serves to reinforce, the above prospects. The upper row of the figure shows the dispersion interactions between two particles: clearly, no passive beam is required for such an observable. The depiction of the pair at the top-left involves both particles in their ground state; as a result the potential energy of the dispersion interaction is always attractive, as has been discussed in Section 4.1. Moving across the diagram, particle $\xi_{1}$ is excited through one-photon absorption (RET from a nearby non-interacting particle is also a plausible source) - enabling the possibility that the pair potential may become repulsive. A process of resonance energy transfer between the pair is then represented on the diagram as 
we move to the right-hand side. The effects on each stage of the process, associated with the throughput of a passive beam with increasing intensity, are indicated in the lower section of the figure. We now calculate the key observables.

First, we focus on the results of single-photon absorption, also indicated by the vertical transition 1 in Fig. 4. Generally, from Eq. (16) with $\xi_{1}$ equivalent to $\xi_{2}$ in all its electronic properties, the change in the dispersion potential produced by this transition is given by:

$$
\Delta E(\alpha, 0)-\Delta E(0,0)=\sum_{s, t}\left(\frac{\boldsymbol{\mu}_{i}^{0 s} V_{i j}(0, \mathbf{R}) \boldsymbol{\mu}_{j}^{0 t} \boldsymbol{\mu}_{k}^{s 0} V_{k l}(0, \mathbf{R}) \boldsymbol{\mu}_{l}^{t 0}}{E_{s 0}+E_{t 0}}-\frac{\boldsymbol{\mu}_{i}^{\alpha s} V_{i j}(0, \mathbf{R}) \boldsymbol{\mu}_{j}^{0 t} \boldsymbol{\mu}_{k}^{s \alpha} V_{k l}(0, \mathbf{R}) \boldsymbol{\mu}_{l}^{t 0}}{E_{s \alpha}+E_{t 0}}\right)
$$

in which $\Delta E(\alpha, 0)$ denotes $\xi_{1}$ in an excited state, i.e. $a=0$ and $b=\beta$ in Eq. (16), and $\Delta E(0,0)$ represents both particles in their ground state $(a=0, b=0)$. In the two-level approximation, the summed states are limited as follows: $s \in\{0, \alpha\}$ and $t \in\{0, \beta\}$.

Since our interest is with a particle pair undergoing essentially irreversible energy transfer, i.e. transfer with a directionality owing to a spectroscopic gradient as indicated earlier, we shall now assume that the electronic excited state $\beta$ of $\xi_{2}$ sits a little lower in energy than the corresponding state $\alpha$ in $\xi_{1}$, from which energy transfers (in the RET process itself, the mismatch is accommodated by internal vibrational relaxation). Accordingly we now write $E_{\beta 0}=E_{\alpha 0}-\delta$, as indicated in Fig. 4, and the previous expression becomes; 


$$
\begin{aligned}
\Delta E(\alpha, 0)-\Delta E(0,0) & =\frac{2\left|\boldsymbol{\mu}^{0 \alpha} \cdot \mathbf{V} \cdot \boldsymbol{\mu}^{00}\right|^{2}}{E_{\alpha 0}}+\left|\boldsymbol{\mu}^{0 \alpha} \cdot \mathbf{V} \cdot \boldsymbol{\mu}^{0 \beta}\right|^{2}\left(\frac{1}{\delta}+\frac{1}{2 E_{\alpha 0}-\delta}\right) \\
& +\frac{1}{E_{\alpha 0}-\delta}\left(\left|\boldsymbol{\mu}^{00} \cdot \mathbf{V} \cdot \boldsymbol{\mu}^{0 \beta}\right|^{2}-\left|\boldsymbol{\mu}^{\alpha \alpha} \cdot \mathbf{V} \cdot \boldsymbol{\mu}^{0 \beta}\right|^{2}\right) .
\end{aligned}
$$

Here, the dependence on the $\mathbf{V}$ tensor is suppressed, and it is evident that the dominant term is given by $\left|\boldsymbol{\mu}^{0 \alpha} \cdot \mathbf{V} \cdot \boldsymbol{\mu}^{0 \beta}\right|^{2} / \delta$. We shall examine the physical implications in more detail below; note for the present that, although the result concerns a shift in static energy, it is the transition dipole moments of the two particles that play the key role in a two-level system.

Now we consider the subsequent process indicated by the coupled transitions 3 and 4 on Fig.

4. The excitation of particle $\xi_{1}$ transfers to $\xi_{2}$ via RET, resulting in the following, further shift in energy between the two relevant configurations. Continuing as before, for a two-level system;

$$
\begin{aligned}
\Delta E(0, \beta)-\Delta E(\alpha, 0) & =\frac{1}{E_{\alpha 0}-\delta}\left(\left|\boldsymbol{\mu}^{00} \cdot \mathbf{V} \cdot \boldsymbol{\mu}^{0 \beta}\right|^{2}+\left|\boldsymbol{\mu}^{\alpha \alpha} \cdot \mathbf{V} \cdot \boldsymbol{\mu}^{0 \beta}\right|^{2}\right) \\
& +\frac{1}{E_{\alpha 0}}\left(\left|\boldsymbol{\mu}^{0 \alpha} \cdot \mathbf{V} \cdot \boldsymbol{\mu}^{00}\right|^{2}+\left|\boldsymbol{\mu}^{0 \alpha} \cdot \mathbf{V} \cdot \boldsymbol{\mu}^{\beta \beta}\right|^{2}\right)-2 \frac{\left|\boldsymbol{\mu}^{0 \alpha} \cdot \mathbf{V} \cdot \boldsymbol{\mu}^{0 \beta}\right|^{2}}{\delta},
\end{aligned}
$$

where, similar to the previous case, it is the term inversely proportional to $\delta$, namely $-2\left|\boldsymbol{\mu}^{0 \alpha} \cdot \mathbf{V} \cdot \boldsymbol{\mu}^{0 \beta}\right|^{2} / \delta$, that is the dominant term. Again the transition dipole moment of each particle proves to be crucial in such a two-level set-up. 
Further insights into the physics are afforded by effecting a rotational average of Eqs (21) and (22) - consistent with Eq. (17) - to deliver results that correspond specifically to electronically isotropic particles. This produces the following;

$\Delta E(\alpha, 0)-\Delta E(0,0)=\frac{1}{24 \pi^{2} \varepsilon_{0}^{2} R^{6}}\left[\frac{2\left|\boldsymbol{\mu}^{0 \alpha}\right|^{2}\left|\boldsymbol{\mu}^{00}\right|^{2}}{E_{\alpha 0}}+\left|\boldsymbol{\mu}^{0 \beta}\right|^{2}\left(\frac{2 E_{\alpha 0}\left|\boldsymbol{\mu}^{0 \alpha}\right|^{2}}{\left(2 E_{\alpha 0}-\delta\right) \delta}+\frac{\left|\boldsymbol{\mu}^{00}\right|^{2}-\left|\boldsymbol{\mu}^{\alpha \alpha}\right|^{2}}{E_{\alpha 0}-\delta}\right)\right]$

$$
\begin{aligned}
\Delta E(0, \beta)-\Delta E(\alpha, 0) & =\frac{1}{24 \pi^{2} \varepsilon_{0}^{2} R^{6}}\left[\left|\boldsymbol{\mu}^{0 \beta}\right|^{2}\left(\frac{\left|\boldsymbol{\mu}^{00}\right|^{2}+\left|\boldsymbol{\mu}^{\alpha \alpha}\right|^{2}}{E_{\alpha 0}-\delta}\right)+\left|\boldsymbol{\mu}^{0 \alpha}\right|^{2}\right. \\
& \left.\times\left(\frac{\left|\boldsymbol{\mu}^{00}\right|^{2}+\left|\boldsymbol{\mu}^{\beta \beta}\right|^{2}}{E_{\alpha 0}}-2 \frac{\left|\boldsymbol{\mu}^{0 \beta}\right|^{2}}{\delta}\right)\right],
\end{aligned}
$$

relating to photo-excitation and RET respectively, in each of which the $R^{-6}$ dependence of the dispersion interaction is now clearly apparent. We shall return to discuss key features of these results in Section 6.

Returning to Fig. 3, the lower row represents configurations with an off-resonant laser beam applied with sufficient intensity. Due to the presence of such a beam, the potentially much larger effect of optical binding may also act on the particle pair, as outlined earlier, but differently for the three illustrated configurations. Moreover, the processes required for the excitations of an interacting particle - i.e. photon absorption and RET in the analyzed diagram are also susceptible to modification by the passive beam; the latter described by the laser- 
assisted resonance energy transfer mechanism. Consider again a rotationally averaged system. As has been shown elsewhere ${ }^{45}$ the effects of optical binding can outweigh those of the dispersion interaction that has been our focus in the above analysis. A crucial difference, associated with a distance-dependence that runs as $R^{-3}$, rather than $R^{-6}$, is a linear dependence on the coupling tensor $\mathbf{V}$. In consequence it becomes evident that, to a first approximation, the passive beam produces no change to the energy shift, for isotropic particles. This is because the corresponding calculation gives a result dependent upon the scalar (weight-zero) part of the coupling tensor, which vanishes in the short range. The considerably more complex calculation that is required for anisotropic systems is to be reported in future work. 


\section{Discussion}

As has been presented, the presence of inter-particle interactions provides two types of phenomena that, although similar in theory, give quite different physical observables: a potential energy or a transfer rate. The former outcome is obtained for interacting permanent dipoles, dispersion interactions and optical binding - where the initial and final system states are identical. Since a transferral of energy necessarily involves differing initial and final states, the experimentally determinable quantity for RET and laser-assisted RET is the transfer rate. Evidence of some confusion over these physical differences is one motivation for this analysis. For example, as recently appeared in the literature, ${ }^{51}$ the notion of an 'RET-induced intermolecular pairing force' is at best a misnomer. In contrast to the delineation of mechanistic interplay that we have described in Section 5, the reported 'new' mechanism could only correspond to an unfeasible merging of optical binding and laser-assisted resonance energy transfer. Although the merging of two phenomena in such a way is not possible, a combination of them is indeed conceivable, as we have shown for dispersion interactions and RET.

Another such combination, which we have pointed to but not fully discussed in the previous text, conflates optical binding and RET. In this prospect, further mechanical motion may arise as the potential energy of optical binding may differ if either (or both) interacting particles are excited via RET or direct absorption. ${ }^{52}$ This contrasts with conventional optical binding, where both particles are normally in their ground state. It should also be stated that the intensity regime of the input laser needs to be around $10^{10}-10^{12} \mathrm{~W} \mathrm{~cm}$-2 for practical observation of mechanical movement or energy transfer modifications; the effects will not be seen at 'room illumination' intensities as offered by ref. [51]. 
A key finding of this research is the discovery that, in the interactions between two molecules in which one has a first excited state slightly lower in energy than its partner, there is an unusually large shift in energy resulting from the excitation of the partner, and also on transfer of electronic excitation from that molecule to the other. These features are exhibited in the terms in Eqs (21) and (22) with an inverse dependence on the energy shift $\delta$. Systems in which marked effects of this kind might be observed could, therefore, be sought in pairs of structurally similar molecules with isolobal electron distributions, but with different chemical substituents for example esters and thio-esters. These are systems in which, despite the changes in the calculated attractive potential due to excitation or energy transfer, there will be little corresponding change in the repulsive interactions from wavefunction overlap. As a consequence of a shift in the intermolecular potential energy minimum, significant changes in equilibrium disposition are anticipated.

In conclusion, we anticipate a variety of potential applications for the tandem operation of optically induced inter-particle couplings, sensor technology based on sub-microscopic motion being a typical example. ${ }^{53}$ As has been shown, this tandem approach may involve a combination of dispersion interactions, optical binding and resonance energy transfer - where the potential energy minimum due to the dispersion interaction is not only modified by optical binding (creating nanoscale motion) but is also affected by excitation of the interacting particles. This, with the prospect of potential applications, forms a backdrop for the undertaking of a number of future studies. 


\section{Acknowledgments}

The authors thank G. Richard Stephenson for insights on the relevance of orbital shape. We are also grateful to the Leverhulme Trust for funding this work.

\section{References}

(1) Cohen, A. E.; Mukamel, S. Phys. Rev. Lett. 2003, 91, 233202.

(2) Obrecht, J. M.; Wild, R. J.; Antezza, M.; Pitaevskii, L. P.; Stringari, S.; Cornell, E. A. Phys. Rev. Lett. 2007, 98, 063201.

(3) Andrews, D. L.; Bradshaw, D. S.; Leeder, J. M.; Rodriguez, J. Phys. Chem. Chem. Phys. 2008, $10,5250-5255$.

(4) Buhmann, S. Y.; Scheel, S. Phys. Rev. Lett. 2008, 100, 253201.

(5) Sherkunov, Y. Phys. Rev. A 2009, 79, 032101.

(6) Rodriguez, J.; Salam, A. J. Chem. Phys. 2010, 133, 164501.

(7) Burns, M. M.; Fournier, J.-M.; Golovchenko, J. A. Phys. Rev. Lett. 1989, 63, 1233-1236.

(8) Mohanty, S. K.; Andrews, J. T.; Gupta, P. K. Opt. Express 2004, 12, 2746-2753.

(9) Bradshaw, D. S.; Andrews, D. L. Phys. Rev. A 2005, 72, 033816.

(10) Dholakia, K.; Zemanek, P. Rev. Mod. Phys. 2010, 82, 1767-1791.

(11) Salam, A. Adv. Quant. Chem. 2011, 62, 1-34.

(12) Lemeshko, M.; Friedrich, B. Mol. Phys. 2012, 110, 1873-1881.

(13) Allcock, P.; Jenkins, R. D.; Andrews, D. L. Phys. Rev. A 2000, 61, 023812.

(14) Andrews, D. L.; Crisp, R. G.; Li, S. P. J. Chem. Phys. 2007, 127, 174702.

(15) Bradshaw, D. S.; Andrews, D. L. J. Chem. Phys. 2008, 128, 144506. 
(16) Craig, D. P.; Thirunamachandran, T. Molecular Quantum Electrodynamics: An Introduction to Radiation-Molecule Interactions; Dover Publications: Mineola, NY, 1998.

(17) Lindgren, I.; Salomonson, S.; Hedendahl, D. Int. J. Quant. Chem. 2008, 108, 2272-2279.

(18) Salam, A. Molecular Quantum Electrodynamics: Long-Range Intermolecular Interactions; Wiley: Hoboken, NJ, 2009.

(19) Woolley, R. G. Proc. R. Soc. London, Ser. A 2000, 456, 1803-1819.

(20) Cohen-Tannoudji, C.; Dupont-Roc, J.; Grynberg, G. Atom-Photon Interactions: Basic Processes and Applications; Wiley: New York, 1992, p. 59.

(21) Louisell, W. H. Quantum Statistical Properties of Radiation; Wiley: New York, 1973.

(22) Capasso, F.; Munday, J. N.; Iannuzzi, D.; Chan, H. B. IEEE J. Sel. Top. Quant. Electron. 2007, 13, 400-414.

(23) Mandel, L.; Wolf, E. Optical Coherence and Quantum Optics; Cambridge University Press: Cambridge, NY, 1995, p. 871.

(24) Daniels, G. J.; Jenkins, R. D.; Bradshaw, D. S.; Andrews, D. L. J. Chem. Phys. 2003, $119,2264-2274$.

(25) Andrews, D. L.; Bradshaw, D. S. Eur. J. Phys. 2004, 25, 845-858.

(26) Clegg, R. M. Fluorescence resonance energy transfer. In Fluorescence Imaging Spectroscopy and Microscopy; Wang, X. F., Herman, B., Eds.; Wiley: New York, 1996; pp 179-251.

(27) Andrews, D. L.; Demidov, A. A. Resonance Energy Transfer; Wiley: Chichester, 1999.

(28) Selvin, P. R. Nat. Struct. Biol. 2000, 7, 730-734.

(29) Scholes, G. D. Annu. Rev. Phys. Chem. 2003, 54, 57-87.

(30) Salam, A. J. Chem. Phys. 2005, 122, 044113.

(31) Sahoo, H. J. Photochem. Photobiol. C 2011, 12, 20-30. 
(32) Andrews, D. L. Can. J. Chem. 2008, 86, 855-870.

(33) Andrews, D. L.; Bradshaw, D. S. Resonance Energy Transfer. In Encyclopedia of Applied Spectroscopy; Andrews, D. L., Ed.; Wiley-VCH: Weinheim, 2009; pp 533-554.

(34) Andrews, D. L.; Juzeliunas, G. J. Chem. Phys. 1991, 95, 5513-5518.

(35) Andrews, D. L.; Rodriguez, J. J. Chem. Phys. 2007, 127, 084509.

(36) Przybytek, M.; Jeziorski, B.; Cencek, W.; Komasa, J.; Mehl, J. B.; Szalewicz, K. Phys. Rev. Lett. 2012, 108, 183201.

(37) Guillon, M.; Moine, O.; Stout, B. Phys. Rev. Lett. 2006, 96, 143902.

(38) Salam, A. Phys. Rev. A 2006, 73, 013406.

(39) Grzegorczyk, T. M.; Kemp, B. A.; Kong, J. A. Phys. Rev. Lett. 2006, 96, 113903.

(40) Karásek, V.; Zemánek, P. J. Opt. A: Pure Appl. Opt. 2007, 9, S215-S220.

(41) Marchington, R. F.; Mazilu, M.; Kuriakose, S.; Garcés-Chávez, V.; Reece, P. J.; Krauss, T. F.; Gu, M.; Dholakia, K. Opt. Express 2008, 16, 3712-3726.

(42) Romero, L. C. D.; Rodríguez, J.; Andrews, D. L. Opt. Commun. 2008, 281, 865-870.

(43) Haefner, D.; Sukhov, S.; Dogariu, A. Phys. Rev. Lett. 2009, 103, 173602.

(44) Taylor, J. M.; Love, G. D. Opt. Express 2009, 17, 15381-15389.

(45) Čižmár, T.; Romero, L. C. D.; Dholakia, K.; Andrews, D. L. J. Phys. B: At. Mol. Opt. Phys. 2010, 43, 102001.

(46) Mellor, C. D.; Fennerty, T. A.; Bain, C. D. Opt. Express 2006, 14, 10079-10088.

(47) Andrews, D. L.; Bradshaw, D. S.; Romero, L. C. D. J. Nanophoton. 2010, 4, 031503.

(48) Andrews, D. L. Phys. Rev. Lett. 2007, 99, 023601.

(49) Bradshaw, D. S.; Andrews, D. L. J. Nanophoton. 2009, 3, 031503.

(50) Bradshaw, D. S.; Andrews, D. L. Superlatt. Microstruct. 2010, 47, 308-313.

(51) Pan, X.; Chen, H.; Wang, W. Z.; Ng, S. C.; Chan-Park, M. B. J. Phys. Chem. A 2011, $115,8155-8166$. 
(52) Andrews, D. L.; Leeder, J. M. J. Chem. Phys. 2009, 130, 034504.

(53) Wang, P.; Pei, H.; Wan, Y.; Li, J.; Zhu, X.; Su, Y.; Fan, C.; Huang, Q. Nanoscale 2012, 4, 6739-6742. 


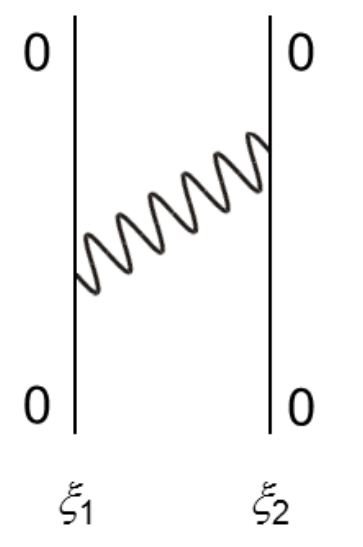

(a)

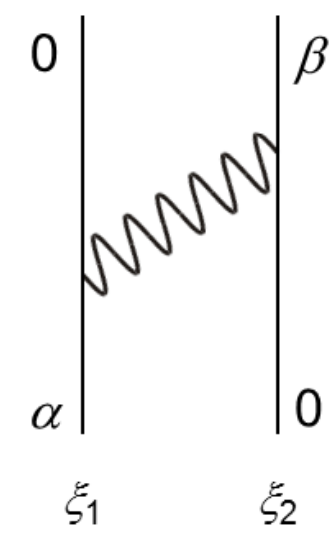

(b)

Fig. 1. Typical Feynman diagrams showing: (a) interacting permanent dipoles, where the initial and final states of each particle (vertical lines) are unexcited, and $(b)$ resonance energy transfer, represented by particle $\xi_{1}$ relaxation from excited state $\alpha$ to ground state 0 and $\xi_{2}$ excitation from 0 to $\beta$. Both observables are mediated by a photon (wavy line), with the intersections denoting particle-light interactions. 


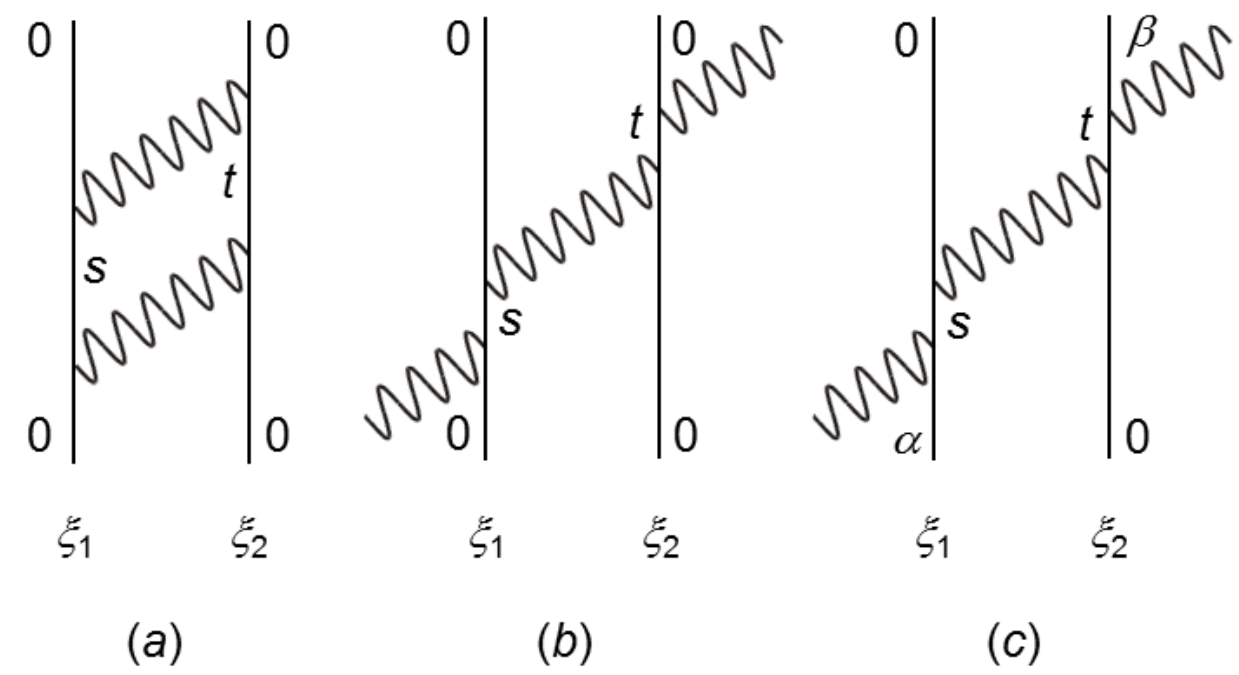

Fig. 2. Typical Feynman diagrams illustrating: $(a)$ the dispersion interactions, where the initial and final states of each particle are unexcited and the intermediate virtual states are denoted by $s$ and $t,(b)$ optical binding, which is essentially identical to the dispersion interactions except a mediating photon between $\xi_{1}$ and $\xi_{2}$ is replaced by an input and an output photon deriving from the passive beam, $(c)$ laser-assisted resonance energy transfer - the construct is similar to optical binding but particle $\xi_{1}$ relaxes from $\alpha$ to 0 and $\xi_{2}$ is excited from 0 to $\beta$. As is conventional, both interactions $(a)$ and $(b)$ have particles which begin and end in their ground states (as shown) but, alternatively, these identical initial and final states may correspond to an excited state. 


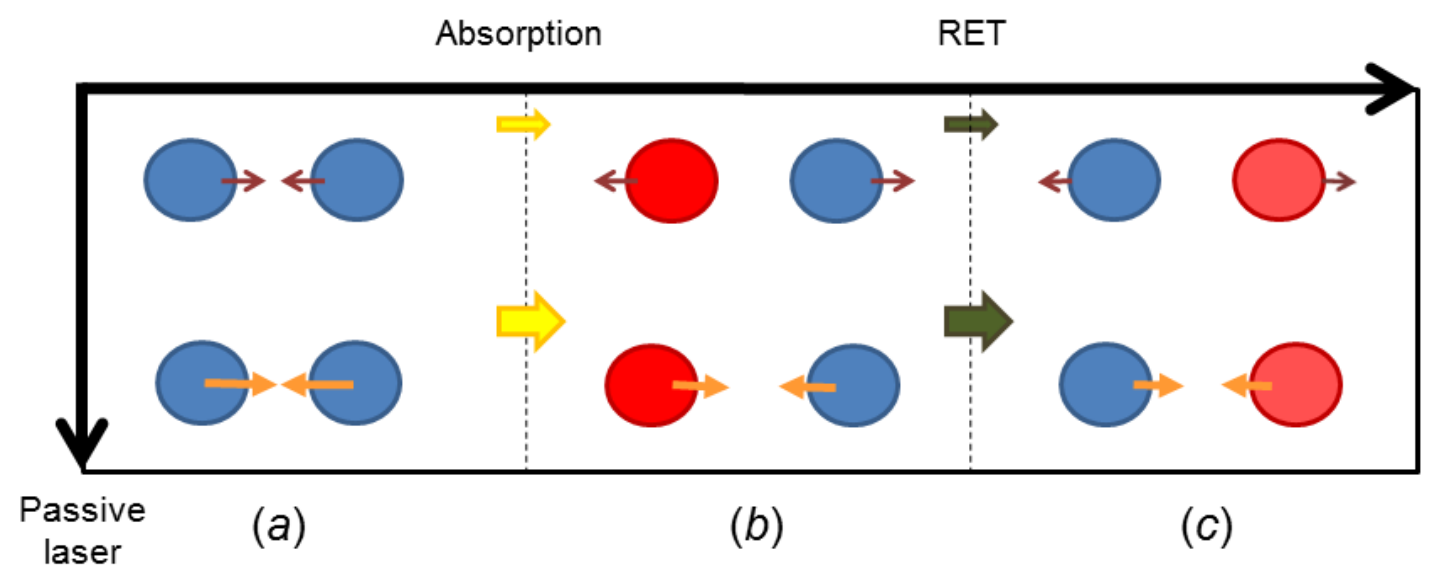

Fig. 3. Representation of the dispersion interaction for: $(a)$ both particles in the ground state (blue circles), (b) particle $\xi_{1}$ in excited state (red circle) with $\xi_{2}$ unexcited, and (c) particle $\xi_{1}$ unexcited and $\xi_{2}$ excited. These states are achieved via the sequential optical process, shown horizontally across the diagram, of one-photon absorption (yellow arrow) followed by resonance energy transfer (green arrow). The purple and orange arrows on the particles denote motion deriving from the dispersion interactions and optical binding, respectively; here the motion is given an arbitrary direction, except the dispersion interactions of unexcited particles which is always attractive. On application of a passive beam of sufficient intensity, denoted by moving vertically down the graph, optical binding may also occur and, additionally, the rate of absorption and energy transfer may be enhanced. 


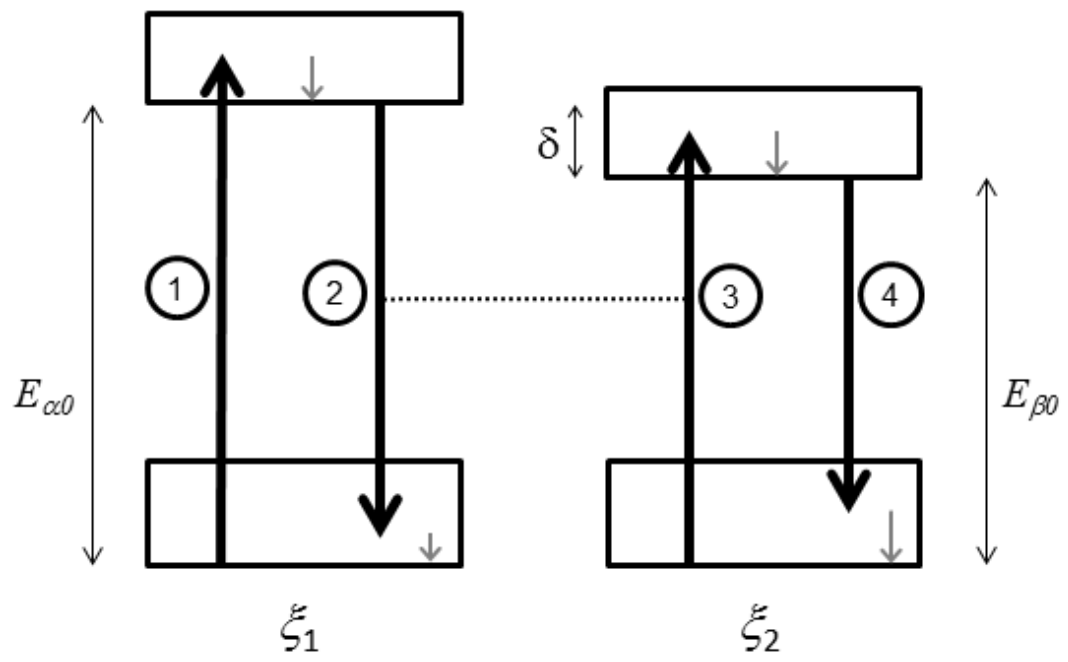

Fig. 4. Jablonski diagram illustrating a sequence of excitation, resonance energy transfer, and emission processes. Arrow (1) represents the excitation of particle $\xi_{1}$ from its initial to final electronic state, followed by intramolecular vibration relaxation (IVR) to the lowest vibration level of its electronic excited state - denoted by the grey arrow. Energy transfer between the two particles is represented by the downward arrow (2) and upward arrow (3), connected by the horizontal dotted line. As before, IVR then occurs within the relevant electronic states. Energy is finally expelled from $\xi_{2}$, shown by arrow (4), and the process is again followed by IVR. The symbol $E_{\alpha 0}$ denotes the energy difference between the lowest vibrational levels of the ground and excited electronic states for $\xi_{1} ; E_{\beta 0}$ is the analogous for $\xi_{2}$ and $\delta$ is the small energy difference between $E_{\alpha 0}$ and $E_{\beta 0}$. 